\title{
Research of Information Model for Power Distribution Equipment Status Management and Control
}

\author{
Shidong Zhang, Linli Zhang, Zhimin Shao, Hejin Liu, Jianxiu Li \\ State Grid Shandong Electric Power Research Institute, Jinan, 250012, China \\ huanazhang@163.com
}

\begin{abstract}
Keywords: Power distribution network equipment; State control; Fault analysis; Distribution network information model.
\end{abstract}

\begin{abstract}
Scope, modeling principle and methods of power distribution network equipment state control model are proposed after research according to actual needs of power distribution network equipment state control. Foundation model applicable to power distribution network equipment state control is tailored on the basis of CIM15 model. Foundation model is corrected, physical equipment and technical parameter attributes are supplemented, manufacturer and product model are added, weather and other external environment models are increased, and information model for supporting power distribution network equipment state control system control and data mining analysis is established. Power distribution network equipment state control model studied in the paper has been applied in constructing power distribution network equipment state control platform.
\end{abstract}

\section{Introduction}

Operation monitoring, defect analysis, fault diagnosis and early warning can be implemented on power equipment in power distribution network on the basis of power equipment grid model data, real-time operation data and historical operation data based on CIM model by combining with faults, defects, test reports and other information in the operation process and integrating external data such as weather condition, etc. Control platform of distribution network equipment status should be constructed, and equipment data of all business systems, existing equipment fault, defect, test report and other data are connected into platform. They are deeply analyzed according to equipment state evaluation, equipment degradation trend analysis, familial defect analysis and other data mining algorithms, thereby realizing distribution network equipment state monitoring, state early warning and familial defect discovery. Failure rate of distribution network equipment can be effectively lowered, maintenance cost of equipment can be reduced, equipment service life can be prolonged, and power grid operation reliability can be improved [1-5].

All business systems of distribution network can be constructed by different manufacturers at different stages according to business requirements. Since different business systems focus on different data gravity centers, different technologies are adopted, and different information models are adopted. Data of all business systems are connected into a unified platform. One integrated database system can be obtained through database extraction, transformation and merge, support can not be provided for senior application of distribution network control, etc. Data of all business systems should be regularly integrated into the platform aiming at integrated equipment monitoring data analysis [6-8]. A unified Distribution Condition Monitoring Model (DCMM) should be established.

DCMM should cover data scope of all business systems on one hand, relationship among all business system data should be established more importantly. Meanwhile, models should be established for weather condition, etc. and associated to related business data model, thereby providing effective support for subsequent equipment state analysis.

In the paper, modeling scope of DCMM, applicable modeling principle and method are firstly determined according to actual demand of distribution network equipment state control on data integration management. Then, information model tailoring process and supplemental content should be described in details, and description is given for supporting control platform construction. 


\section{DCMM modeling scope}

Comprehensive data of equipment should be analyzed aiming at distribution network equipment status control. The following data are required: (1) physical equipment data, including physical equipment standing book, manufacturer model, state overhaul and other information, should be obtained mainly through e production management system (PMS); (2) Operation equipment data should be obtained by topological structure among equipment described from the perspective of operation through distribution network automation system (DAS), energy management system (EMS) and GIS system; (3) Power grid real-time data, equipment operation voltage, current, load and other data; (4) Equipment defects, fault, test report and other data; (5) External environment data, such as weather data, etc. Data required for covering the above distribution network equipment state control analysis is required during DCMM modeling.

\section{DCMM construction}

IEC 61970 and IEC 61968 Common Information Model (CIM) are very applicable to be used as foundation of DCMM tailoring expansion as information model defined according to international standard in power system management and information exchange fields[10].

After modeling scope of DCMM is determined, CIM model is firstly tailored to form CIM foundation model. Modeling guide for CIM modeling is adopted. Types, properties and correlations for describing monitoring point, physical equipment, technical parameter, test report and other abstract description required by distribution network equipment state control should be expanded and established. Unified Modeling Language (UML) consistent with CIM model should be adopted as modeling language.

\section{Foundation model construction}

CIM model released CIM10 in 2003, and it is the first widely used stable version. CIM10 is widely applied in EMS, and other system, and CIM16v17 was released in 2013 through constant development and upgrading. CIM version change speed is fast, which may lead to some troubles in practical application. CIM versions are numerous, which version shall be adopted? In general, the latest CIM version should be adopted as far as possible to ensure conformance with standard CIM model. However, the latest version tends to be unstable. In practice, stable new version is adopted as benchmark. A part of modes of other versions should be suitably integrated according to business needs. Modes from CIM and expansion in different versions should be distinguished through different namespace.

DCMM chooses CIM15v33 as benchmark version based on the above consideration. All mode element namespace from the benchmark version is shown as follows: http://iec.ch/TC57/2011/CIM-schema-cim15\#. Mode element namespace expanded according to CIM14 version is shown as follows: http://iec.ch/TC57/2009/CIM-schema-cim14\#. Mode element namespace expanded and established according to business need is shown as follows: http://yjy.sd.sgcc.com.cn/2015/DCMM. Use of namespace faciliates mode combination, DCMM can be updated and expanded according to CIM in different versions according to need of distribution grid equipment state control.

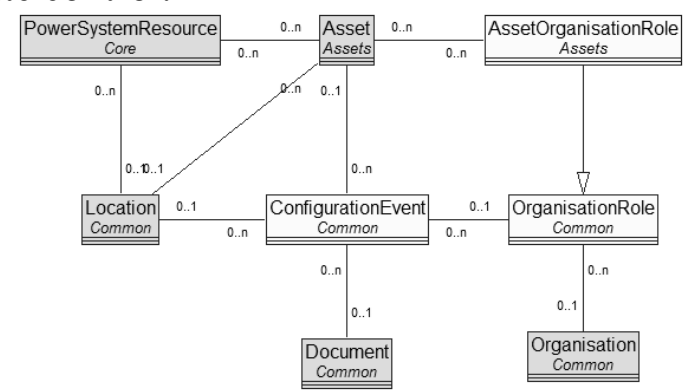

Fig.1. Foundation Model of DCMM

Foundation models include operation equipment, physical equipment, location, document, organization, etc. Five systems of power system resource, asset, location, document and 
organization, and sub-class thereof in TC57 CIM are selected to form foundation model. Power system resource system is used for describing logical structure, network topology; Asset is direct target of state control; location is used for describing concrete location, GIS coordinates; document is used for describing defect, test report and other records; organization is used for describing power supply bureau and all manufacturers(figure 1).

\section{Foundation model fix}

Direct correlation is discovered among power system resource, asset and location in foundation model. Correlation is established through middle role asset organization role between asset and organization as shown in figure 2 .

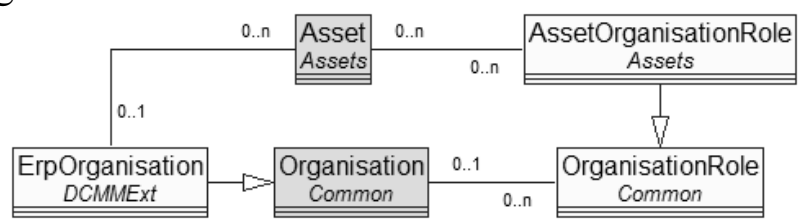

Fig.2. Fix Base Model

The modeling mode is adopted for solving the condition that the same asset is correlated with the same organization for many times. For example, one department acts as commanding department, maintenance department and use department of assets. However, the modeling mode is more troublesome during actual use. Commanding department of assets should be searched twice. In addition, different correlations can be distinguished through using attribute value of asset organization role, and semantic representation is not very clear. in order to solve the above problems, the most common asset commanding department of DCMM during modeling is represented by direct correlation Asset-ErpOrganization. Original mode is still retained for expressing other correlation and expansion. Similarly, self-correlation of assets is used for representing asset hierarchy. Self-correlation of organization represents organization hierarchy. Document self-correlation is used for representing document hierarchy; Document-asset association represents the relation between defect document record and concrete physical equipment. Document - organization association represents document department.

\section{Physical equipment extension}

Physical equipment is modeled as subclass of asset in DCMM. DCMM is expanded on the basis of CIM model, and subclass treatment is implemented on physical equipment. SubstationAsset, CircuitAsset, BreakerAsset, CableAsset,OverheadConductorAsset and other specific subclass of asset are established. From asset association of assets is adopted for representing the superior physical equipment.

General expansion mode is adopted for CIM model aiming at technical parameters of physical equipment. UserAttribute class is used for representing technical parameters as shown in figure 3 (a). The mode has the maximum flexibility, but it has too strong randomness in actual use with insufficient semantic constraints. The string type is adopted value type, which is not conducive to subsequent analysis and other applications. Therefore, technology parameters are modeled according to different categories during DCMM modeling. Technical parameters with some electric features should be directly regarded as attributes in assets category for modeling. Description class should be established and associated to assets object aiming at some statistical technical parameters.,figure 3(b).UserAttribute mode is adopted for constructing some technical parameters with explanation features and subsequent expansion technical parameters.

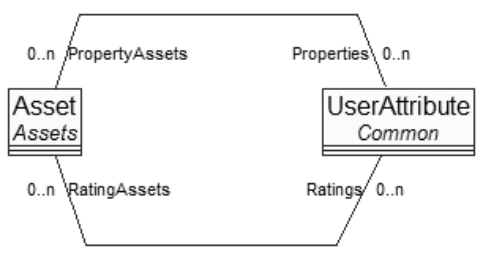

(a)

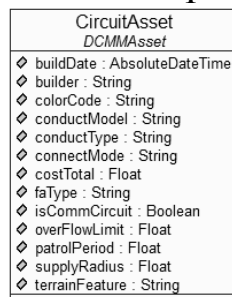
$\$$ supplyRadius : Float
$\diamond$ terrainFeature : String

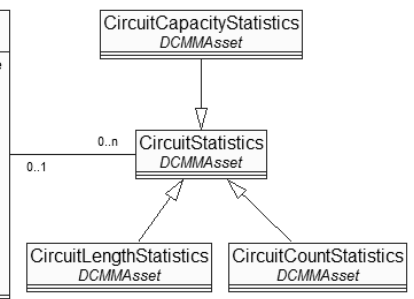

(b)

Fig.3. Technical Parameter Model 


\section{Circuit extension}

Circuit belongs to more important equipment in power distribution network. In DCMM, circuit equipment is expressed by CircuitAsset class. Circuit is composed of conductor assets in sections, which are divided into two classes, namely OverheadConductorAssets on the ground and underground CableAsset.

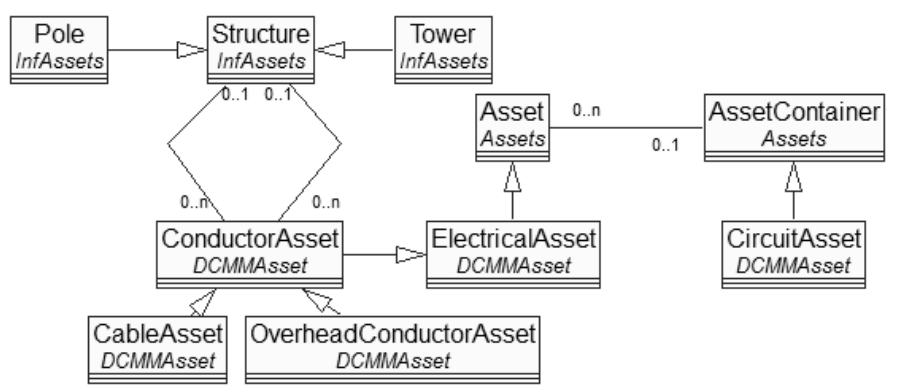

Fig.4. Circuit Model

Pole foundation structure establishes two association relationships with conductor asset in order to describe starting pole and ending pole of conductor, which are used for representing starting and ending locations of conductor(figure 4)

\section{Asset evaluation}

Asset evaluation refers that equipment is scored according to certain method. It is foundation to analyze equipment health state trend. AssetEvaluation is a subclass of document in DCMM. Evaluated equipment is expressed through associating document and asset(figure 5).

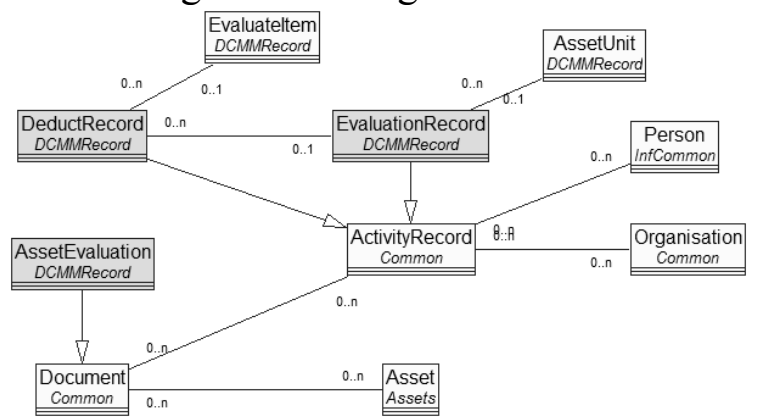

Fig.5. Asset Evaluation Model

Asset evaluation consists of a set of detailed evaluation record. Evaluation record modeling belongs to subclass of activity record. Evaluation record is associated with a group of deduct record, detailed deduct cause are recorded in record evaluation.

\section{Weather monitoring extension}

Weather monitoring data belongs to external environment data. Data come from timing monitoring of weather station. Weather station location is fixed, which is associated with substation. All Circuit, switches and other physical equipment from substation share monitoring data of one weather station(figure 6).

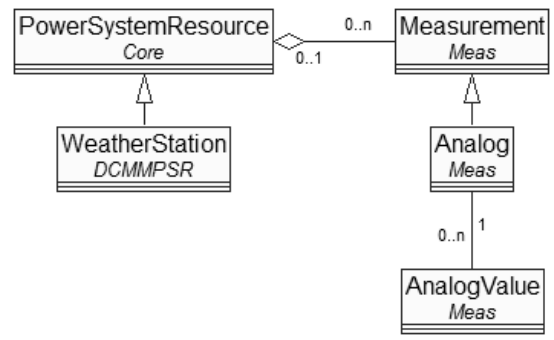

Fig.6. Weather Model

Weather monitoring data includes wind speed, wind direction, temperature, humidity, rainfall, visibility, air pressure, wind speed, wind direction, maximal wind speed and maximal wind direction. Analog and AnalogValue object are respectively established, and their association with whether station is established.

Concrete weather station and monitoring data of weather station are established in model data aiming at weather monitoring data. Weather data of detailed time sequence are read and written 
through historical data access interface.

\section{Model and manufacturer extension}

Model and manufacturer is also a more important part in distribution network equipment state control analysis, and it is foundation of familial defect analysis.

When physical equipment is designed, required physical equipment type asset can be set. Types can be catalogued. Type refers to some technical parameter set required in design. Concrete physical equipment mode must be in line with type request(figure 7).

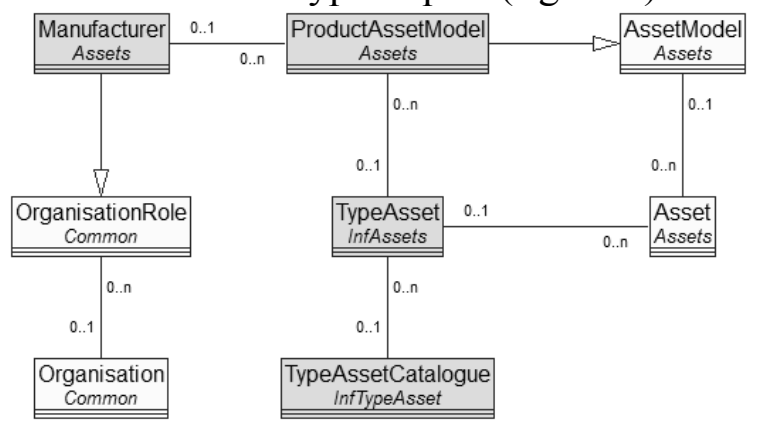

Fig.7. AssetModel Model

Manufacturer manufactures product asset model. When concrete physical equipment is selected, the type must be in line with type requirement. Concrete type is associated with physical equipment, and support is provided for analysis of familial defects.

Manufacturer is a subclass of organization role, and a role played by concrete organization. Detailed organization information is described in associated organization. Manufacturer is modeled into organization role rather than being directly modeled into a subclass of organization. The scene that one organization acts as many roles in actual condition is solved. For example, one organization is regarded as supplier, maintainer or electricity consumer. The modeling mode can reduce creation of repeated organization object.

\section{DCMM support for control platform}

DCMM model restricts business semantically from the top layer. Models of all business systems are integrated. Associations of data in various business systems are established, independent data of various systems are integrated into integral data. Data is standardized in the data access process, barriers among various business systems are solved, thereby providing effective support for data mining and data analysis.

DCMM model is adopted to drive development of the following key technologies:

\section{Model mapping}

Since current mainstream storage system is still a relational database, DCMM model is established by object-oriented mode, the mapping relationship between object orientation and relational database should be established.

Object-oriented mode is also adopted for IEC 61970 CIS interface [11]. When data is accessed through CIS interface, conversion between object orientation and relational database can be achieved through object-relational mapping algorithm. When data is read through CIS interface, data in relational database can be converted into object data. When data is written through CIS interface, the object can be converted into data in relational database.

\section{View definition}

A network object relation is established by CIM model. However, similar tree-shaped hierarchical structure is adopted for visit during actual business use. Tree-shaped definition should be constructed according to DCMM model, and data can be provided outwards in the form of tree-shaped hierarchical structure.

DCMM model is expanded and modified constantly, dynamic definition of tree-shaped hierarchical structure and exhibition in dynamic tree shape are focus keys.

\section{Standardized release}

Normalized DCMM model only acts as platform foundation. Normalized data should be released 
outwards in the platform. In addition, standardized model, real-time, historical and event data release institutions are also required. Standardized IEC 61970 CIS service interface should be utilized for releasing data.

\section{Conclusion}

DCMM is abstractly established through models related to distribution network equipment state control. Control platform is established on the basis of DCMM. Scattered data of all business systems is integrated into integrated unified panoramic view data. Intrinsic value of existing data can be better explored. Data standardization and redundancy removal are basically realized through control platform established by combining with standardized CIS interface based on DCMM model. Existing data are effectively integrated, thereby providing effective data for fault analysis and other algorithms. Models related to equipment state control are constantly supplemented to DCMM model with continuous development of equipment state control. Continuous development of control platform and constant extension of application analysis are supported.

\section{References}

[1] WANG Bai-jun, ZHOU Yi-hua, REN Xiao-ying. Research and Implementation on Fault Intelligent Monitoring Technology of Distribution Circuit [J]. China Electric Power (Technology Edition), 2013, (12), pp.1-3(in Chinese).

[2] GUAN Long, LIU Zhi-gang, XU Jian-fang, et al. Key issues with model-based diagnosis in distribution network[J]. Power System Protection and Control, 2012, 40(20), pp.145-150(in Chinese).

[3] NI Qiong, YU Tao, HE Bin-bin. The study of distribution network equipment status and risk assessment. Electronic Test, 2014, (24) , pp.116-118(in Chinese).

[4] Gao Zhen-xing, Guo Chuang-xin, YU Bin, et al. Study of a fault diagnosis approach for power grid with information fusion based on multi-data resouces[J]. Power System Protection and Control, 2011, 39(6), pp.17-23(in Chinese).

[5] LI Peng, ZHANG Xiaoyi, HUANG Haosheng, et al. Research on Power Network Fault Diagnosis System with Lightning Location Information [J]. Jiangsu Electrical Engineering, 2014, 33(2), pp.17-21(in Chinese).

[6] CHEN Fen. Research on the Method of Integrating Data Based on Several Different Electric Automation Systems [J]. Mechanical \& Electrical Engineering Technology, 2014,(1) , pp.70-72(in Chinese).

[7] ZHANG Zhisheng, CAO Ming, GAO Shangfei, at el. Research on Transmission Panoramic Cockpit Technology Based on Data Fusion of Grid Maintenance and Nanagement Data [J]. Yunnan Electric Power, 2014, (5) , pp.114-117(in Chinese).

[8] XU Yan, ZHANG Rui, WEI Bo. Novel Fault Diagnosis Approach of Power Grid Using Data Fusion [J]. Proceedings of the CSU-EPSA, 2014, 26(11), pp.67-71(in Chinese).

[9] IEC 61970-501 energy management system application program interface (EMS-API), part 501:Common Information Model Resource Description Framework (CIM RDF) schema[S]. IEC, 2006-05.

[10] IEC 61970-301 energy management system application program interface (EMS-API), part 301: common information model (CIM) base[S], Edition 2.0. IEC, 2009-04.

[11] IEC 61970-403 Energy management system application program interface (EMS-API) Part 403: Generic data access [S], Edition 1.0. IEC, 2008-06. 